\title{
A interpretação de cognições e emoções com o conceito de eventos privados e a abordagem analítico-comportamental da ansiedade e da depressão
}
The interpretation of cognitions and emotions with the concept of private events and the behavior-analytic approach to anxiety and depression

\section{Darlene Cardoso Ferreira, Liany Tavares Tadaiesky, Nilzabeth Leite Coêlho, Simone Neno e Emmanuel Zagury Tourinho ${ }^{1}$} [1] Universidade Federal do Pará (UFPA), Brasil | Título abreviado: Eventos privados | Endereço para correspondência: Av. Tavares Bastos, 438,
Apto 204. CEP: 66613140. Belém, PA. I E-mail: darlene.cardoso@gmail.com | Nota: Trabalho parcialmente financiado pela CAPES (Bolsa
de Mestrado), FAPESPA (Bolsa de Mestrado) e CNPq (Processos 304116/2007-5 e 480727/2007-4).

\begin{abstract}
Resumo: Cognições e emoções constituem um tema básico para qualquer sistema explicativo na psicologia. $\mathrm{Na}$ análise do comportamento, são tratadas como fenômenos comportamentais e abordadas com o auxílio do conceito de eventos privados. Segundo Skinner, eventos privados são estímulos e respostas inacessíveis a uma observação pública direta. O presente artigo revisa alguns usos do conceito de eventos privados na análise do comportamento, aponta controvérsias e limites no uso do conceito e relaciona a esses usos interpretações analítico-comportamentais da depressão e da ansiedade. O texto fundamenta-se especialmente em literatura analítico-comportamental que enfatiza que cognições e emoções são relações comportamentais, com graus variados de complexidade, das quais apenas alguns termos são estímulos privados ou respostas encobertas. Finalmente, o texto aponta algumas implicações para a prática do terapeuta analítico-comportamental e a necessidade de desenvolvimento de tecnologias baseadas em competências acadêmicas e profissionais de analistas do comportamento.
\end{abstract}

Palavras-chave: eventos privados, terapia analítico-comportamental, ansiedade, depressão

\begin{abstract}
Cognitions and emotions constitute a basic issue in every explanatory system in psychology. In behavior analysis, cognitions and emotions are viewed as behavioral phenomena, and are approached with the concept of private events. According to Skinner, private events are stimuli and responses which are inaccessible to direct public observation. This paper reviews the Skinnerian approach to private events, some controversies and limits in the use of the concept, and illustrates its use in the analysis of depression and anxiety. The paper is specially committed to a behavior-analytic literature which emphasizes that cognitions and emotions are behavioral relations, with variable degrees of complexity, of which some terms are private stimuli or covert responses. The paper points out some implications to the practice of behavior-analytic therapists, and the need to develop technologies directed to intervention, based on behavior analysts' academic and professional competences.
\end{abstract}

Keywords: private events, behavior-analytic therapy, anxiety, depression 
Cognições e emoções constituem um tema básico para qualquer sistema explicativo na psicologia. $\mathrm{Na}$ análise do comportamento, são tratadas como fenômenos comportamentais, contrastando com modelos interpretativos que as consideram ocorrências em outro domínio de análise e mesmo dotados de uma natureza (imaterial) distinta daquela dos fenômenos comportamentais. A afirmação da natureza comportamental de cognições e emoções requer, porém, uma sofisticada elaboração conceitual, que se apóia tanto em evidências empíricas sobre o comportamento humano, quanto em análises conceituais ou filosóficas que nos remetem ao exame crítico da linguagem das emoções.

O conceito de eventos privados está no centro das explicações analítico-comportamentais para cognições e emoções. O presente artigo revê a proposição do conceito de eventos privados por Skinner (1945) e alguns usos do conceito na literatura da área. A esses usos o artigo relaciona algumas interpretações analítico-comportamentais de fenômenos referidos como depressão e ansiedade. Por último, o artigo sugere algumas direções para a pesquisa clínica na análise do comportamento, baseadas na apropriação da elaboração disponível sobre eventos privados. Destacam-se nesta revisão as noções de que: (a) "eventos privados" consistem de uma resposta verbal emitida sob controle de eventos/fenômenos diversos (o que lhe confere certa imprecisão); e (b) conceitos emocionais são respostas verbais emitidas sob controle de fenômenos comportamentais de complexidade variável.

\section{O Conceito de Eventos Privados}

Ao introduzir o conceito de eventos privados, Skinner (1945) aponta a possibilidade (e a necessidade) de a psicologia comportamental lidar com temas relacionados à subjetividade, como pensamentos, emoções e cognições, sem o apelo a uma natureza imaterial dos fenômenos. Esta vem a ser uma característica distintiva de sua filosofia behaviorista radical, para a qual, no lugar de abandonar o estudo da subjetividade, compete a uma ciência do comportamento analisá-la como fenômeno comportamental.

De um modo geral, eventos privados são tidos como estímulos e respostas não acessíveis à observação pública direta, mas acessíveis diretamen- te ao próprio indivíduo a quem dizem respeito. Cognições e emoções poderiam ser analisadas, segundo essa ótica, como relações comportamentais que se diferenciam simplesmente porque pelo menos um estímulo ou uma resposta que participa da relação é privado, isto é, não pode ser diretamente observado pelos outros. Uma resposta privada, ou encoberta, envolve uma reduzida participação do aparelho motor, daí sua observabilidade reduzida. Um estímulo privado é localizado no próprio corpo do indivíduo e, em geral, afeta-o de um modo único, como não pode afetar outros indivíduos.

Pelo menos três razões justificariam o interesse da análise do comportamento pelo estudo da subjetividade (Tourinho, 1999a). A primeira seria sua própria relevância para a definição do campo de estudos da psicologia, na medida em que a cultura ocidental, particularmente, estimula os indivíduos a se comportarem de forma "introspectiva", ensinaos a relatar sentimentos como causas de seus comportamentos e a comportar-se discriminativamente sob controle destas falas.

Dada a importância atribuída a tais repertórios autodescritivos, uma segunda razão estaria relacionada ao fato de, muitas vezes, as autodescrições ficarem sob controle parcial de estímulos privados, de maneira que uma análise funcional poderia indicar os modos particulares como os indivíduos interagem com suas alterações corporais e como, nesta interação, vai definindo-se sua privacidade.

Por fim, novamente como resultado de certas práticas culturais, alguns contextos de aplicação da análise do comportamento demandam um tipo de intervenção que contemple os eventos privados. A terapia clínica analítico-comportamental, por exemplo, requer do analista do comportamento clínico interpretações acerca do comportamento verbal descritivo de emoções, sentimentos e pensamentos (Tourinho, 2006a). Desta forma, à análise do comportamento cabe desenvolver modos de atender às demandas produzidas pela cultura sem, contudo, reproduzir o mentalismo por ela veiculado (Tourinho, 1997c, 1999b).

Para Anderson, Hawkins e Scotti (1997), a despeito da relevância do tema, pouco foi produzido acerca do conceito de eventos privados desde a proposição de Skinner (1945). Segundo os autores, o ritmo consideravelmente lento de produção de co- 
nhecimento sobre eventos privados na análise do comportamento explica, em parte, o ecletismo e as incertezas dominantes na terapia comportamental.

Como já mencionado, ao discutir o conceito de eventos privados, Skinner (1945, 1953/1965, 1974/1976) faz referência a estímulos privados e respostas encobertas de um indivíduo, aos quais somente ele teria acesso direto. Portanto, apenas a observabilidade diferenciaria esses eventos: "um evento privado pode distinguir-se por sua acessibilidade limitada, mas, até onde sabemos, não por qualquer estrutura ou natureza especial" (Skinner, 1953/1965, p. 257). Skinner (1974/1976) afirma ainda: "uma pequena parte do universo está contida dentro da pele de cada um de nós. Não há razão porque devesse ter qualquer status especial apenas por localizar-se entre estes limites" (p. 24). Esta referência a uma fronteira definida pela pele não pretende implicar uma identificação do "privado" com o "interno", mas apenas o reconhecimento de que, eventualmente, uma condição corporal pode adquirir a função de estímulo (cf. Tourinho, 2006c) e de que isso é o que acontece quando falamos de estímulos privados. Por essa razão, segundo Tourinho (1999b, 2006b), associações entre privado e interno devem ser tratadas com cautela. Nem todo evento corporal é um estímulo (seja ele público ou privado) e nem todo estímulo corporal (e interno) é um estímulo privado. Por exemplo, um nervo inflamado pode ser um estímulo (visual) público, assim como pode ser um estímulo (interoceptivo) privado, um não implicando o outro. Nesse sentido, o que define a privacidade não é a localização do evento, e a pele não constitui necessariamente uma fronteira. Da mesma forma, a dicotomia interno-externo não é útil para caracterizar respostas, embora algumas sejam abertas e outras encobertas. Isto é, não faz sentido falar em respostas internas ou externas, mas pode-se considerar que algumas respostas são inacessíveis à observação pública (Tourinho, 1999b).

Quando se considera a privacidade como problema psicológico, não se pretende focalizar as alterações fisiológicas de um organismo, posto que as estruturas e funções físico-químicas constitutivas da base biológica dos fenômenos comportamentais enquadram-se no campo investigativo de outras disciplinas científicas. Ao se considerar eventos corporais na análise do comportamento, importa descobrir como os indivíduos reagem às condições corporais e a maneira pela qual isto participa do controle de comportamentos públicos (Tourinho, 1997a). Caberia, então, uma distinção entre o privado como condições corporais, domínio da fisiologia, e como eventos com função comportamental, objeto de estudo da análise do comportamento.

Eventos inobserváveis e internos, a exemplo de alterações anátomo-fisiológicas, podem tornar-se parte constitutiva do fenômeno comportamental de interesse da psicologia, adquirindo funções de estímulo não por si mesmas, mas apenas como membros de uma relação de contingência que envolve elementos publicamente observáveis.

A aprendizagem comportamental, promovida pelas contingências sociais às quais o indivíduo é exposto, tem papel essencial no que concerne a cognições e emoções, que se caracterizam como relações comportamentais que envolvem respostas emitidas encobertamente pelo indivíduo, ou respostas públicas parcialmente sob controle de estímulos privados. Contudo, qualquer resposta encoberta está apenas circunstancialmente encoberta, não sendo naturalmente inacessível à observação pública.

Algumas razões que explicam a forma encoberta de emissão de uma resposta operante são apontadas por Skinner (1957/1992, 1974/1976). Respostas encobertas podem ser produto de um controle de estímulos fraco, insuficiente para uma emissão pública da resposta; o ambiente apresentaria, então, somente uma parte do estímulo discriminativo que normalmente ocasionaria a resposta em sua forma pública (Skinner, 1957/1992). Outro fator estaria relacionado à maior conveniência e facilidade, num determinado contexto, de emitir-se uma resposta encoberta, o que aumentaria sua probabilidade de ocorrência em detrimento da forma publicamente observável (Skinner, 1957/1992, 1974/1976).

Ainda mais relevante, segundo Skinner (1957/1992, 1974/1976), seria a exposição do organismo a contingências sociais, envolvendo consequências punitivas contingentes à emissão aberta de algumas respostas. Atividades do organismo como o pensar, às quais geralmente se atribui uma natureza especial, constituem, simplesmente, respostas adquiridas e emitidas inicialmente na forma pública. Estas respostas teriam retrocedido a um nível enco- 
berto em função das contingências de reforçamento adicionais - em geral, a punição social contingente à emissão da resposta na forma aberta.

Sentimentos também são frequentemente apontados, no senso comum, como causas de comportamentos. À luz dos pressupostos analítico-comportamentais, porém, "sentir" constitui uma resposta do organismo como um todo, cujas relações funcionais com eventos externos devem ser identificadas da mesma forma como se buscam as causas de respostas publicamente observáveis.

Conforme Skinner (1945), somente ao interagir com uma comunidade verbal é que o indivíduo poderá aprender a responder discriminativamente sob controle de certas condições corporais. Deste modo, por exemplo, um conjunto indiferenciado de alterações fisiológicas só será identificado pelo indivíduo como "alegria", "felicidade", "depressão" ou "ansiedade" após a aquisição de um repertório verbal autodescritivo, promovido por uma comunidade que observa os indivíduos e os ensina a descreverem suas sensações de acordo com os correlatos públicos aos quais têm acesso. Segundo Tourinho (1999b), apenas quando se vive numa sociedade na qual se é constantemente indagado a respeito do que se sente, é que se adquirem comportamentos descritivos de sentimentos.

Como a comunidade tem acesso somente a eventos públicos ao ensinar repertórios autodescritivos, é impossível afirmar se a resposta do indivíduo ficou sob controle de uma condição interna precisa. Tourinho (2006a) ressalta, contudo, que o importante não é se uma descrição de sentimento é mais ou menos precisa ou correspondente a uma condição anátomo-fisiológica particular, e sim enfatizar que respostas verbais autodescritivas, como "estou ansioso", podem ficar sob controle de estímulos privados equivalentes ou correlacionados com estímulos públicos.

Para o sujeito, a resposta autodescritiva pode ficar parcialmente sob controle dessa condição corporal associada ao correlato público, de modo que esta adquirirá função de estímulo, tornando-se parte de uma contingência. Por outro lado, se um indivíduo pode emitir uma resposta sob controle de uma condição corporal, ao emiti-la está sob controle de uma correspondência entre esta condição e os eventos nos quais a sociedade baseia seu reforçamento. Assim, nenhuma condição corporal pode exercer autonomamente o controle discriminativo de uma resposta verbal, já que somente contingências sociais ensinam indivíduos a reagir de um modo particular às suas condições corporais, e tanto as contingências quanto os modos de reação são verbais (Tourinho, 1997a,1997c).

Em razão da dependência do comportamento verbal de contingências sociais, o controle de respostas autodescritivas não se desvincula das contingências baseadas em estímulos públicos, mesmo quando a resposta é emitida sob controle de estímulos privados associados. Além disso, o controle eventual por um estímulo privado só é possível na medida em que o repertório verbal é mantido pela ação reforçadora da comunidade verbal. Em síntese, os conceitos utilizados para descrever fenômenos supostamente privados são respostas verbais pelo menos parcialmente sob controle de eventos públicos (Tourinho, 2006a).

Skinner (1945) também chama a atenção para o fato de a comunidade verbal exercer um papel importante, cujo alcance vai além da aquisição das respostas verbais. Mesmo quando o repertório autodescritivo foi estabelecido, a ação reforçadora desta comunidade será baseada na correspondência entre o relato e as respostas associadas publicamente observáveis (Tourinho 1997a, 1997c). Isto é, o papel de contingências públicas não se encerra com a aquisição da resposta verbal autodescritiva. Não é verdade que, uma vez tendo aprendido a descrever-se como deprimido - parcialmente sob controle de uma estimulação privada -, a ocorrência posterior da resposta é função apenas dessa condição. Ela continua sendo função (também) de estímulos públicos.

\section{Usos da Dicotomia Público-Privado na Literatura da Análise do Comportamento}

Alguns modos como os eventos privados costumam ser discutidos na análise do comportamento podem ser problemáticos. A caracterização de pensamentos, emoções e sentimentos como "comportamentos que ocorrem sob a pele" pode implicar suposições diferentes. Algumas vezes esta expressão pode referir-se a eventos privados enquanto ocorrências internas; outras vezes refere-se à inacessibilidade destes à observação pública. Todavia, como apon- 
tado antes, pode ser incorreto falar de uma correspondência entre privado e interno, já que eventos considerados internos nem sempre são inacessíveis à observação pública, assim como eventos inacessíveis à observação pública nem sempre são internos (Tourinho, 1997d, 1999a). A noção de interioridade dos eventos privados costuma estar relacionada à suposição de que eventos privados coincidem com condições anátomo-fisiológicas do organismo. Sabe-se, entretanto, que as condições corporais, tomadas isoladamente, sem qualquer função comportamental, não são objeto de estudo da psicologia. Além disso, não existe comportamento "sob a pele”. Toda resposta é uma resposta do organismo como um todo (Tourinho, 1997b, 1997d, 1999a). Ainda, todo fenômeno comportamental consiste de uma relação da qual participam vários eventos, com função de estímulo ou de resposta. Isso vale para pensamentos e sentimentos, que não são eventos discretos, mas relações ou conjuntos de relações comportamentais.

No contexto de uma ciência do comportamento, é mais adequado discutir eventos privados sob a ótica da inacessibilidade pública. A formulação da dicotomia público-privado enfatiza a impossibilidade de observação direta por outros indivíduos dos eventos considerados privados. Apesar disso, deve-se reconhecer que mesmo a referência à inacessibilidade à observação pública direta pode dar origem a controvérsias na análise do comportamento. Diversos comportamentos inacessíveis à observação púbica não podem ser considerados como pertinentes para uma discussão acerca da subjetividade humana. Por exemplo, digitar uma senha no caixa de um banco é um evento inacessível à observação pública direta, porém dificilmente poderíamos considerá-lo como uma questão de ordem subjetiva.

Tourinho (2006a) ressalta que a elaboração skinneriana do conceito de eventos privados teve como objetivo discutir os "termos psicológicos" de acordo com os postulados analítico-comportamentais. Isto é, é importante ter clareza sobre quais fenômenos estamos tentando compreender com o conceito de eventos privados. Basicamente, com o conceito de eventos privados estamos focalizando estímulos e respostas que não são observáveis aos outros ou porque estão localizados no corpo do próprio indivíduo que estabelece com estes um contato diferente daquele possível a outros observadores, ou porque envolvem uma participação restrita do aparelho motor, no caso das respostas encobertas (Tourinho, 2006b).

No que se refere aos estímulos privados, podese falar em dois tipos de estimulações localizadas no próprio corpo do indivíduo: a interoceptiva e a proprioceptiva. O sistema interoceptivo compreende as estimulações derivadas dos sistemas digestivo, respiratório e circulatório; já o sistema proprioceptivo inclui as estimulações relativas aos músculos, tendões, juntas, etc. (Skinner, 1974/1976).

Existe ainda um terceiro sistema nervoso apontado por Skinner (1974/1976), o exteroceptivo, por meio do qual o indivíduo entra em contato com a estimulação presente no ambiente circundante. Estímulos exteroceptivos (i.e., visuais, olfativos, táteis, auditivos e gustativos), mesmo que localizados no corpo do indivíduo que responde a eles, não devem ser considerados privados, pois podem afetar outras pessoas além do próprio indivíduo.

Por sua vez, uma condição corporal que afeta um indivíduo sob a forma de estimulação privada (e.g., interoceptiva) também pode afetar outros indivíduos, sob a forma de estimulações exteroceptivas. Nesse caso, não estamos diante do mesmo estímulo, mas da mesma alteração corporal, que tem diferentes funções de estímulo para o próprio indivíduo e para os outros. Por exemplo, o indivíduo pode emitir a resposta verbal "lesão no músculo" sob controle de um estímulo proprioceptivo, enquanto observadores externos poderão emitir esta resposta verbal sob controle do mesmo evento, mas que os afeta como estímulo exteroceptivo (e.g., a visualização do inchaço na região muscular).

Um evento privado pode assumir diversas funções em uma relação comportamental, como a de eliciador incondicionado ou condicionado, estímulo reforçador, discriminativo ou estabelecedor. Quando se fala da função discriminativa de um estímulo privado para uma resposta verbal autodescritiva, estamos diante de processos frequentemente denominados de autoconhecimento. Para Skinner (1945), é a comunidade verbal quem dispõe as contingências necessárias para a instalação de respostas autodescritivas dos eventos privados. Respostas verbais descritivas de eventos privados são adqui- 
ridas sob controle de um conjunto de estímulos, incluindo estímulos públicos, que participam das contingências dispostas pela comunidade verbal (Skinner, 1945, 1974/1976). Portanto, a funcionalidade de uma resposta autodescritiva depende da correlação com as contingências públicas, embora esta resposta possa estar também parcialmente sob controle de estímulos privados (cf. Tourinho, 1997a, 1997d, 2006b).

Em relação às respostas encobertas, é necessário estabelecer parâmetros que as diferenciem como pertinentes à temática da subjetividade daquelas cuja inobservabilidade decorre de barreiras físicas entre observador e observado, não sendo resultado de contingências sociais que definem sua forma de emissão. Respostas encobertas não são encobertas por natureza; as contingências sociais exercem um papel decisivo para a emissão de uma resposta de modo aberto ou encoberto. Nesse caso, segundo Skinner (1953/1965), a resposta encoberta ocorre em uma escala tão reduzida que não pode ser observada por outros sem um aparato instrumental adequado. Tourinho (2006b) propõe a análise das propriedades formais e relacionais da resposta como critério para definir seu caráter encoberto, conforme será abordado em seguida.

Ao discutir o responder verbal encoberto, Skinner (1957/1992) aponta a existência de uma ordem decrescente de "energia" da resposta verbal, que seria afetada por condições especiais de reforço. Para o autor,

A gama de comportamentos verbais consiste, aproximadamente, numa ordem decrescente de energia, em gritar, falar alto, falar baixo, sussurrar, resmungar, falar subaudível com uma ação muscular detectável, falar subaudível de dimensões pouco claras e talvez até mesmo o 'pensar inconsciente', inferido algumas vezes em exemplos de solução de problemas. Nesse continuum, não há um ponto no qual seja proveitoso traçar uma linha distinguindo pensamento e ação. (p. 521)

Tourinho (2006b) propõe que isto seja interpretado como uma referência a graus variados de observabilidade das respostas conforme o nível de participação do aparelho motor do indivíduo. Neste sentido, respostas verbais encobertas poderiam ser caracterizadas como respostas verbais emitidas com baixos níveis de participação da musculatura vocal de um indivíduo.

Entretanto, não é necessário estabelecer uma fronteira entre respostas abertas e encobertas. Ao invés disso, pode-se supor que as formas de emissão de uma resposta variam em um continuum, que vai da maior à menor observabilidade. Esta variação na observabilidade é que depende tanto de aspectos formais da resposta (o grau de participação do aparelho motor) quanto de aspectos da relação entre observador e observado.

Skinner (1953/1965, 1974/1976) afirma que todas as respostas são inicialmente aprendidas na forma aberta, passando a ser emitidas aberta ou encobertamente conforme as contingências sociais em vigor. Deste modo, a forma de emissão de uma resposta não é devida à sua natureza, e sim aos estímulos ambientais presentes. Tourinho (2006b) articula as proposições de alguns autores (Donahoe \& Palmer, 1994; Hayes, 1994; Kantor \& Smith, 1975; Observer, 1973, 1981) e sugere outras variáveis para discutir o nível de observabilidade das respostas.

O treino dos observadores pode ser considerado uma primeira variável que influencia o grau de observabilidade de certas respostas. Um observador bem treinado teria mais facilidade de identificar algumas respostas que poderiam ser tidas como encobertas por outro observador não treinado (Tourinho, 2006b). Por exemplo, um observador treinado para identificar os sinais não verbais característicos do comportamento de mentir terá mais facilidade de perceber quando alguém está mentindo do que aquele observador não treinado.

Outra fonte de variabilidade do nível de observabilidade de uma resposta pode ser encontrada na argumentação de Donahoe e Palmer (1994) acerca da importância dos instrumentos do observador. Neste sentido, haveria uma relação direta e proporcional entre os instrumentos do observador e a possibilidade de identificação das respostas do observado.

A familiaridade ou intimidade entre observador e observado pode ser considerada outra variável que afeta o nível de observabilidade de uma resposta (cf. Hayes, 1994). Tourinho (2006b) sugere que estas proposições da abordagem de Kantor po- 
dem ser utilizadas em uma interpretação analíticocomportamental das respostas encobertas. Deste modo, um observador que possui uma história de vida compartilhada com um indivíduo terá maior probabilidade de identificar suas respostas do que um observador que acabou de ser apresentado a ele. Neste caso, a observabilidade da resposta não seria função apenas de uma propriedade formal, mas também de dimensões relacionais.

Tourinho (2006a, 2006b) sugere, então, a articulação das abordagens expostas (Donahoe \& Palmer, 1994; Hayes, 1994; Kantor \& Smith, 1975; Observer, 1973, 1981; Skinner, 1953/1965, 1974/1976), definindo-se a observabilidade de respostas como uma função de suas propriedades formais/estruturais e de suas propriedades relacionais. As propriedades formais e estruturais da resposta estão relacionadas às suas características topográficas, ou seja, ao grau de participação do aparelho motor na emissão da resposta. Já as propriedades relacionais referem-se à influência de algumas variáveis na definição do nível de observabilidade das respostas, como treino do observador, instrumentos do observador e grau de familiaridade entre observador e observado. Tourinho (2006b) aponta ainda a compatibilidade desta noção com a argumentação de Skinner sobre a ordem decrescente de energia das respostas verbais e o papel das contingências sociais para a definição de respostas encobertas.

De modo análogo, pode-se falar de um continuum de observabilidade dos arranjos de estímulos que controlam respostas autodescritivas de emoções e sentimentos. Respostas verbais autodescritivas não estão sob controle de estímulos privados apenas, existindo uma relação necessária destes estímulos com eventos públicos. Assim, quanto maior a participação de eventos públicos no controle das respostas autodescritivas, maior o nível de observabilidade.

Esta elaboração teórica acerca de um continuum de observabilidade a partir das propriedades formais e relacionais das respostas é diferente de uma visão tradicional de eventos enquanto públicos ou privados - que, de certo modo, reproduz a lógica dualista criticada pela abordagem skinneriana. Falar de comportamentos como abertos ou encobertos ignora a premissa de que o comportamento é sempre uma relação complexa da qual podem participar estímulos e respostas públicos e privados. Assim, o comportamento não pode ser definido como público ou privado, visto que em uma relação comportamental os estímulos privados e as respostas encobertas são apenas termos da contingência.

Além disso, partindo-se da noção de um continuum de observabilidade, estímulos e respostas não devem ser classificados como públicos ou privados, mas sim como eventos que variam nesse continuum em virtude de suas propriedades formais e relacionais. Conforme Tourinho (2006a), "observabilidade ou inobservabilidade não constituem propriedades absolutas de estímulos e respostas; são pólos de um continuum ao longo do qual variam certos eventos em contextos de relações" (p. 120).

Outra limitação da dicotomia público-privado consiste da diversidade de definições para eventos privados, oferecidas por analistas do comportamento. Tourinho (2006a, 2006b, 2007) destaca que o uso diverso do conceito de eventos privados está relacionado ao enfoque de fenômenos diferentes, como condições anátomo-fisiológicas, eventos constitutivos de relações comportamentais e relações comportamentais propriamente ditas.

Quando analistas do comportamento afirmam que eventos privados podem ser produtos colaterais das contingências (e.g., Skinner, 1974/1976), compreende-se que esta resposta verbal se dá sob controle das condições anátomo-fisiológicas envolvidas no fenômeno. Todavia, estas condições são produtos de uma história genética e ambiental e não se confundem com o produto comportamental. No momento em que elas passam a ser consideradas "causas do comportamento", elas já terão adquirido a função de estímulos públicos ou privados (cf. Tourinho, 2006b). Quando o uso do conceito trata dos eventos constitutivos de relações comportamentais, a referência é feita a estímulos privados e respostas encobertas. Contudo, conforme já discutido, estímulos e respostas são termos de uma contingência e partes de uma relação comportamental, não se caracterizando como comportamentos em si. Por fim, quando eventos privados são definidos como relações comportamentais, deve-se levar em consideração que estas relações são relações de contingências, envolvendo necessariamente termos observáveis que nem sempre podem ser caracterizados como produtos colaterais das contingências. 
As relações comportamentais envolvidas nos fenômenos cognitivos e emocionais abrangem fenômenos com graus variados de complexidade, que têm em comum a presença de um estímulo privado ou uma resposta encoberta como um dos termos da contingência. Logo, eventos privados participam de fenômenos constituídos por poucas ou diversas relações comportamentais, verbais, não verbais ou ambas. De acordo com Tourinho (2007), "se essa complexidade é tão variável, então talvez não seja possível ao terapeuta, por exemplo, definir antecipadamente como um evento privado pode ou deve entrar em sua análise ou seu objeto de intervenção" (p. 6), pois isto dependerá da função que o evento privado exerce na contingência analisada. Neste sentido, a compreensão de fenômenos comportamentais considerados pertinentes à privacidade ou subjetividade a partir de sua localização ao longo de um continuum de complexidade pode ser relevante.

\section{Eventos Privados e Relações Comportamentais com Graus Variáveis de Complexidade}

De acordo com o modelo de complexidade proposto por Tourinho (2006a, 2006b), sentimentos e emoções - definidos enquanto fenômenos psicológicos ou comportamentais que envolvem eventos privados - podem ser compreendidos como relações (ou conjuntos de relações) comportamentais. A partir da noção skinneriana de comportamento humano como produto de contingências filogenéticas, ontogenéticas e culturais (Skinner, 1953/1965), assume-se que eventos comportamentais humanos podem incluir relações produzidas por variáveis de um ou mais destes níveis. As relações que participariam de um dado fenômeno comportamental determinariam o grau de complexidade deste fenômeno, em um continuum de complexidade (Tourinho, 2006b).

O modelo de complexidade proposto por Tourinho (2006b) sugere que fenômenos comportamentais são mais ou menos complexos dependendo do conjunto de relações envolvidas. Comportamentos mais complexos envolveriam relações determinadas por variáveis dos três níveis filogenético, ontogenético e cultural. Já fenômenos menos complexos incluiriam relações compostas por variáveis somente do primeiro e do segundo ní- veis $^{1}$. O continuum de complexidade apresentaria, assim, duas extremidades. Em uma delas, assinala Tourinho (2006a), localizar-se-iam comportamentos determinados por variáveis da filogênese - em que algumas respostas ficaram sob controle de eventos cuja função de estímulo foi adquirida ao longo da história evolutiva do homem. Na outra extremidade, estariam os fenômenos constituídos por redes de relações estabelecidas nos três níveis de variação e seleção. Em pontos intermediários, encontrar-seiam eventos comportamentais definidos em termos de relações mais ou menos complexas.

Conforme a proposta em questão, complexidade indica uma noção de inclusividade, na medida em que fenômenos comportamentais mais complexos incluem relações formadas por variáveis dos níveis anteriores (Tourinho, 2006a). Assim, um dado comportamento pode ser considerado mais complexo por envolver contingências dos três níveis, e não somente relações culturalmente determinadas, por exemplo.

Vale ainda ressaltar que mesmo fenômenos localizados nos limites de um mesmo nível podem diferir quanto ao grau de complexidade, dependendo das relações que envolvem (Tourinho, 2006a, 2006b). Assim, é possível que dois eventos comportamentais, ambos constituídos por variáveis filogenéticas, ontogenéticas e culturais, localizemse em intervalos distintos do continuum, embora estejam no segmento de maior complexidade. Por exemplo, a resposta de um indivíduo de escolher uma sobremesa menos calórica após a refeição, por ter aprendido que sobremesas menos calóricas são mais saudáveis, certamente envolve contingências compostas por variáveis dos três níveis de seleção. Contudo, esse comportamento apresenta um nível de complexidade inferior se comparado ao de um indivíduo que, a despeito de um peso corporal re-

1 Tourinho (2006a) ressalta que dificilmente encontraríamos um evento comportamental envolvendo apenas variáveis do primeiro nível. Não obstante, haveria comportamentos fortemente determinados pelo nível filogenético, os quais, embora pudessem incluir relações ontogeneticamente determinadas, estariam mais próximos da extremidade do continuum composta por fenômenos de menor complexidade. A título de exemplo, podemos citar respostas em que os componentes fisiológicos são essenciais para sua ocorrência, como salivar diante do alimento. 
duzido, evita ingerir refeições muito calóricas porque vê a si como uma pessoa obesa - característica comum entre pessoas que apresentam transtornos alimentares como anorexia. Nesse último caso, embora também estejam presentes variáveis filogenéticas, ontogenéticas e culturais, estas apresentam uma configuração que provê maior complexidade ao fenômeno comportamental.

O modelo de complexidade é útil para discutir-se uma apropriação consistente do conceito de eventos privados, na medida em que chama a atenção para o fato de que estímulos privados e respostas encobertas podem ser parte de fenômenos comportamentais humanos diversos em muitos aspectos, sobretudo na quantidade e nos tipos das relações que os definem. Deste ponto de vista, sentimentos e emoções - ou quaisquer outros fenômenos aos quais se atribui relação com o componente da privacidade - apresentam graus diferenciados de complexidade.

No caso de fenômenos dos quais participam variáveis dos três níveis de seleção, o grau mais elevado de complexidade não decorre somente de processos seletivos repetidos, mas também dos tipos de variáveis envolvidas no controle (Tourinho, 2006a). Isto se aplica especialmente no que tange à inclusão de contingências verbais que tornam possíveis novas redes de relações.

Segundo Friman, Hayes e Wilson (1998), as respostas autodiscriminativas (de caráter necessariamente verbal) de um indivíduo podem afetar seu comportamento de diferentes modos. Para os autores, mais do que descrever comportamentos e circunstâncias, relatos podem modificar a função de respostas e de contingências descritas. Desta forma, repertórios autodescritivos, de auto-observação ou mesmo respostas controladas por autodescrições, podem passar a constituir fenômenos comportamentais diversos. Mais ainda, enquanto componentes de uma relação, podem adquirir função de estímulo para outras respostas. Em um contexto terapêutico, por exemplo, deve-se compreender qual a função das verbalizações do indivíduo, na medida em que podem controlar outros comportamentos. Tendo em vista as relações possíveis entre descrições de sentimentos e outros comportamentos, o papel do terapeuta seria identificar as situações nas quais seria necessário rom- per os vínculos estabelecidos pela transferência de funções entre estímulos. O fenômeno comportamental, pois, deve ser analisado em termos de todas as redes de relações que o definem.

A noção de fenômenos comportamentais - inclusive os que envolvem eventos privados - com diferentes graus de complexidade possibilita, como também requer, um refinamento e diferenciação dos modos de se tratar estes fenômenos tanto em âmbito conceitual quanto clínico. Assim, por exemplo, se a tristeza de um indivíduo se define principalmente por comportamentos operantes não verbais, relacionados à redução de apetite e diminuição da frequência de sair de casa, as intervenções terapêuticas podem privilegiar a análise de contingências não verbais relevantes, sem necessariamente dar grande atenção a componentes verbais. No entanto, se o fenômeno envolve predominantemente relações verbais, como pensamentos suicidas, autorregras depreciativas, entre outras, será necessário privilegiar essas relações na intervenção. Nesse último caso, a exposição direta a contingências não verbais, por exemplo, não seria suficiente para modificar o fenômeno.

\section{Ansiedade e Depressão como Fenômenos dos quais Participam Eventos Privados}

Nossa sociedade costuma definir como ansiedade ou depressão repertórios comportamentais apresentados em situações bem distintas. Assim, diz-se que um indivíduo possui problemas de ansiedade quando se declara incompetente para falar em público apesar de ter vasto repertório verbal e, do mesmo modo, caracteriza-se como ansioso um indivíduo que, sem qualquer razão clara para isso, não consegue sair de casa para visitar um amigo. Ansiedade e depressão podem constituir exemplos de conceitos que abarcam fenômenos comportamentais com graus de complexidade diferentes.

Partindo, portanto, do pressuposto de que ansiedade e depressão apresentam-se como fenômenos comportamentais complexos que podem ser compreendidos como redes de relações entrelaçadas, variando em um continuum de complexidade, é possível abordar cada fenômeno tentando identificar seus componentes respondentes, operantes não verbais e operantes verbais. 


\section{Ansiedade, depressão e a relação com componentes fisiológicos}

No que diz respeito ao papel das respostas fisiológicas na definição de depressão e ansiedade, a discussão varia em torno de dois aspectos principais: condições anátomo-fisiológicas como subproduto de contingências específicas, principalmente contingências aversivas, e a possibilidade de tal condição fisiológica participar dessas relações adquirindo alguma função.

No primeiro caso, tratando-se da ansiedade, alguns autores focalizam especificamente a exposição do organismo a estímulos aversivos e pré-aversivos, controláveis ou incontroláveis, como sendo responsável por produzir uma condição fisiológica particular, concomitante a uma mudança no responder geral do organismo (Forsyth, 2000; Forsyth \& Eifert, 1996a, 1996b; Oliveira \& Duarte, 2004; Queiroz \& Guilhardi, 2001; Reiss, Peterson, Gursky \& McNally, 1986; Wolpe, 1981).

Outro aspecto apresentado por alguns autores (e.g., Bornstein, 1975; Santos, 2000; Wlazlo, Hartwing, Hand, Kaiser \& Münchau, 1990; Wolpe, 1981) é a noção de que a condição fisiológica pode ser tratada como um mero "sintoma" de um fenômeno que, nesse caso, é necessariamente outra coisa (envolvendo outros tipos de relações comportamentais).

Com relação à depressão, os componentes fisiológicos são analisados por Dougher e Hackbert (1994), a partir da idéia de eliciação incondicionada, na qual contingências relacionadas à punição e extinção ou à disponibilidade insuficiente de reforço funcionariam como estímulos incondicionados capazes de gerar subprodutos emocionais característicos da depressão. Os autores ressaltam também a possibilidade de eliciação condicionada, na qual alguns estímulos discriminativos associados à punição ou à inacessibilidade de reforçamento poderiam adquirir função eliciadora respondente condicionada.

Levando em consideração a análise referente à possibilidade de componentes orgânicos participarem de relações adquirindo alguma função, essa condição fisiológica característica do fenômeno denominado de ansiedade, por exemplo, pode ser vista como: (a) um estímulo discriminativo para respostas verbais ou não verbais (Forsyth, 2000; Forsyth
\& Eifert, 1996a; Forsyth, Eifert \& Thompson, 1996; Wolpe \& Rowan; Zvolensky, Lejuez \& Eifert, 1998); (b) um estímulo discriminativo para respostas não verbais mantidas por reforço negativo (Bouton, Mineka \& Barlow, 2001; Zamignani \& Banaco, 2005); e (c) um estímulo discriminativo para respostas não verbais mantidas por reforço positivo, considerando, por exemplo, a possibilidade da condição corporal funcionar como estímulo discriminativo para respostas que produzam atenção ou algum tipo de reforço social positivo (Zamignani \& Banaco, 2005; Zamignani \& Vermes, 2003).

Outro ponto a ser destacado é que tanto na ansiedade quanto na depressão as próprias reações emocionais (fisiológicas) podem adquirir função aversiva em algum momento (Dougher \& Hackbert, 1994; Skinner, 1953/1965).

\section{O papel dos componentes operantes verbais e não verbais na análise da ansiedade e da depressão}

No que se refere aos componentes operantes não verbais e operantes verbais envolvidos na depressão e na ansiedade, algumas análises são necessárias. No tocante às relações operantes não verbais, têm-se várias possíveis condições relacionadas a funções dos estímulos envolvidos. Por exemplo, eventos (privados ou públicos) correlacionados com extinção ou punição podem gerar comportamento de fuga $\mathrm{e}$ esquiva com a função de estímulos discriminativos específicos. Ou ainda, eventos relacionados às consequências envolvidas poderiam adquirir função de consequências mantenedoras de padrões de resposta características da depressão (Cavalcante, 1997; Dougher \& Hackbert, 1994; Hunziker, 2005; Lejuez, Hopko \& Hopko, 2001; Seligman, 1975/1992). Além disso, o modelo do desamparo aprendido sugere que a relação de um estímulo com a incontrolabilidade de uma situação (Seligman, 1975/1992) pode também evocar o padrão (de inatividade) típico na condição de incontrolabilidade.

Desta forma, destacam-se algumas das relações comportamentais que podem integrar quadros de ansiedade. São elas: (a) supressão condicionada (Forsyth, 2000; Oliveira \& Duarte, 2004; Queiroz \& Guilhardi, 2001; Sanger \& Blackman, 1976; Silva, 1997; Viliers \& Millenson, 1972; Zamignani \& Banaco, 2005); (b) respostas de fuga e/ou esquiva 
da estimulação aversiva (Barbosa, 2004; Bouton, Mineka \& Barlow, 2001; Cone, 1998; Eifert, 1984; Eifert \& Wilson, 1991; Forsyth, 2000; Friman, Wilson \& Hayes, 1998; Hopko, McNaiel, Zvolensky \& Eifert, 2001; Jones \& Friman, 1999; Lejuez, O'Donnell, Wirth, Zvolensky, \& Eifert, 1998; Oliveira \& Duarte, 2004; Öst, 1987; Reiss, Peterson, Gursky \& McNally, 1986; Santos, 2000; Silva, 1997; Zamignani \& Banaco, 2005; Zamignani \& Vermes, 2003; Wlazlo, Hartwing, Hand, Kaiser \& Münchau, 1990; Wolpe, 1981) e (c) a impossibilidade de controle sobre a apresentação da estimulação aversiva (incontrolabilidade) (Barbosa, 2004; Forsyth \& Eifert, 1996a; Zvolensky, Lejuez \& Eifert, 1998).

Ainda com relação à ansiedade, Woods e Miltenberger (1996) e Zamignani e Banaco (2005) destacam algumas operações de reforçamento, como, por exemplo, a eliminação de outros estímulos que não o estímulo aversivo, ou seja, a retirada ou o adiamento de uma tarefa indesejável como importante na caracterização da ansiedade. Ademais, a manutenção de respostas de ansiedade por reforçamento positivo, principalmente na forma de atenção e de outros reforçadores sociais contingentes, também são destacadas por Zamignani e Banaco (2005) e Zamignani e Vermes (2003).

Outro aspecto relacionado aos componentes operantes não verbais dos fenômenos de ansiedade e depressão diz respeito a operações estabelecedoras, nas quais certos eventos produzem a mudança da efetividade reforçadora de outros eventos, tendo como resultado possível: (a) aumento do efeito reforçador de certas consequências; (b) aumento da probabilidade de respostas que tenham produzido reforços estabelecidos no passado e (c) aumento da efetividade evocativa de estímulos discriminativos associados a reforços estabelecidos. Neste caso, os eventos associados com o estado característico da depressão potencializam algumas contingências e despotencializam outras (Dougher \& Hackbert, 1994).

Já na ansiedade, pode haver estímulos verbais, como "cognições errôneas", apontadas por Reiss, Peterson, Gursky e McNally (1986); Wlazlo, Hartwing, Hand, Kaiser e Münchau (1990) e Wolpe (1981), ou ainda interpretações catastróficas quanto aos sintomas fisiológicos sentidos, assinalados por Torres (2000), como eventos verbais capazes de tor- nar o indivíduo mais vulnerável a sentir-se ansioso. Além disso, Zamignani e Banaco (2005) destacam a possibilidade de contingências ambientais aversivas, que levam o indivíduo a uma condição crônica de interações sociais, bem como do estado de privação poderem ser vistos enquanto operações estabelecedoras para o padrão característico da ansiedade.

Outro ponto a ser levado em consideração é a análise das possibilidades de operantes verbais (principalmente autorregras) participarem do controle de respostas verbais identificadas como depressão e ansiedade. Nesse sentido, o condicionamento semântico ${ }^{2}$ destacado por Bouton, Mineka e Barlow (2001), Eifert (1984), Forsyth e Eifert (1996b), Forsyth, Eifert e Thompson (1996), Tyndall, Roche e James (2004) e Weiss e Evans (1978) e a possibilidade de equivalência de estímulos apontada por Forsyth e Eifert (1996b), Tyndall, Roche e James (2004) e Zamignani e Banaco (2005), no caso da ansiedade, podem ser tidos como possibilidades de expansão para um outro nível de complexidade na análise do fenômeno.

Da mesma forma podem ser analisadas as discussões apresentadas por Dougher e Hackbert (1994) a respeito de mando ou mandos estendidos na forma de autocrítica, tatos distorcidos (autorregras) que podem funcionar como potencializadores de contingências depressivas ou até mesmo estímulos verbais que podem adquirir função aversiva devido à sua participação em relações de equivalência de estímulos.

Hunziker (2001) propõe, neste caso, que, no desamparo aprendido, o indivíduo poderia formular regras que descrevessem a discriminação quanto ao seu poder de controlar ou não os eventos do meio. Neste ponto, ela afirma que

os relatos de pacientes deprimidos (do tipo 'eu sei que não adianta fazer nada, pois minha vida é mesmo uma droga, nada vai mudar') seriam expressões dessas regras que controlam seu comportamento. Nesse sentido, o desamparo

2 Segundo Forsyth e Eifert (1996b), o condicionamento semântico seria o processo pelo qual algumas palavras adquirem função de controle sobre respostas emocionais. Para eles, "um estímulo palavra, pareado com um estímulo incondicionado (SI) que elicia uma resposta emocional, também virá a eliciar a resposta emocional" (p. 614). 
aprendido seria um exemplo de comportamento governado por regras. (p. 146)

\section{Uma análise dos fenômenos ansiedade e depressão: intervenções possíveis}

Pensar em ansiedade e depressão como fenômenos complexos dos quais participam eventos privados é pensar sob a lógica de que, dependendo de quais os componentes envolvidos, cada instância desses fenômenos localiza-se em um ponto do continuum de complexidade abordado anteriormente. Como afirma Tourinho (2006b),

conceitos tais como "depressão" e "ansiedade" usualmente referem-se a um conjunto de relações que estão de alguma maneira conectadas; por exemplo, padrões de interação social, repertórios de auto-observação, outras respostas públicas sob controle de autodescrições etc. $\mathrm{O}$ que liga todos esse fenômenos ao campo da privacidade é o fato de que para cada um deles há relações das quais uma estimulação privada ou uma resposta encoberta podem fazer parte. Estes não são produtos colaterais de contingências de reforçamento, fenômenos inteiramente inobserváveis, ou simplesmente uma questão de uma contingência de três termos. (p. 24)

É importante destacar que este tipo de análise repercute diretamente nas propostas de intervenção frente a fenômenos descritos como depressão e ansiedade. Levando em conta o modelo apresentado por Tourinho (2006b), pode-se interpretar os diferentes tipos de intervenção como possibilidades de alcance da terapia analítico-comportamental. Sendo assim, se entendermos os fenômenos de ansiedade e depressão como fenômenos comportamentais com diferentes níveis de complexidade, as intervenções propostas devem focalizar-se nas diferentes relações presentes na definição dos fenômenos.

Em alguns casos, intervenções baseadas em condições operantes não verbais, como a exposição ao evento temido, no caso de ansiedade, podem ser eficazes nos casos em que estes fenômenos incluem relações dos níveis filogenético e ontogenético. Entretanto, quando relações operantes verbais passam a constituir o foco das relações envolvidas, com a necessidade de aumento de repertórios de habi- lidades sociais por meio de treino de habilidades sociais, por exemplo, no caso da depressão (Kanter, Callaghan, Landes, Busch \& Brown, 2004), estratégias de intervenção que priorizem estas relações talvez sejam necessárias. Como afirma Tourinho (2006b), "o terapeuta deve trabalhar para alterar essas funções de estímulo das respostas verbais sobre outros repertórios do indivíduo" (p. 29).

Não se trata, portanto, de afirmar que uma intervenção é mais eficaz do que outra, mas de chamar a atenção para o fato de que é necessário intervir sobre as relações que estão envolvidas, sendo elas dos níveis filogenético, ontogenético ou cultural. Como destaca Tourinho (2006b), "perguntar sobre aqueles componentes ou relações comportamentais pode funcionar como uma estratégia conceitual, esclarecendo as classes de eventos com os quais nós podemos ter que lidar e uma estratégia de intervenção, esclarecendo as relações que precisam ser mudadas" (p. 29). A escolha da intervenção mais apropriada vai depender de uma análise de cada um desses níveis e das possibilidades de entrelaçamento entre as relações encontradas.

Esta nova possibilidade de interpretação de cada um dos fenômenos aqui referidos como exemplos deve ser um ponto de partida para pesquisas na área clínica voltadas a aspectos pouco esclarecidos. Coêlho (2006) aponta uma agenda de pesquisas na seguinte direção: (a) ansiedade e a relação com eventos reforçadores positivos ou com repertórios de autocontrole; (b) aspectos relacionados à incontrolabilidade do evento aversivo com sinalização pré-aversiva, como na ansiedade, ou sem a sinalização pré-aversiva, como é o caso da depressão. Além disso, pesquisas relacionadas a uma relação de previsibilidade (ansiedade) ou imprevisibilidade (depressão) também devem ser realizadas, pois as implicações para a intervenção em cada um destes casos merecem ainda ser examinadas.

\section{Considerações Finais}

Com o conceito de eventos privados, a análise do comportamento permite-se abordar fenômenos tidos como subjetivos, reconhecendo-lhes propriedades especiais, mas evitando a lógica dualista com que tradicionalmente foram abordados na psicologia. A proposição do conceito por Skinner (1945), porém, precisa ser vista como um ponto 
de partida e não como um ponto de chegada para a análise dos fenômenos complexos que definem nossas cognições e emoções.

Se a (in)observabilidade de estímulos e respostas constitui a referência principal para compreender a especificidade de cognições e emoções, é importante ter em conta que essa (in)observabilidade decorre não de propriedades espaciais, nem exclusivamente de propriedades formais, mas depende sobretudo de aspectos relacionais. Compreender a lógica relacional do pensamento analítico-comportamental é, assim, essencial para apreender sua efetiva contribuição no campo do estudo das emoções e cognições.

Quando se avança na demarcação dos usos possíveis e consistentes do conceito de eventos privados para a análise da subjetividade, chega-se ao reconhecimento de que olhar para estímulos privados ou respostas encobertas é fundamental, porém absolutamente insuficiente para dar conta dos fenômenos de interesse. Tais fenômenos transcendem em muito as ocorrências discretas (estímulos e respostas) para as quais somos levados a atentar com o conceito de eventos privados e são conformados por relações ou redes de relações que tornam a tarefa de análise muito mais exigente, refinada e abrangente. Avanços oferecidos nessa direção pela literatura da análise do comportamento podem mostrar-se uma ferramenta de grande utilidade ao terapeuta analítico-comportamental, tanto do ponto de vista conceitual quanto da atividade prática cotidiana.

A possibilidade de analisarmos cada conceito da linguagem das emoções e das cognições com o modelo de interpretação descrito ao longo deste artigo requer atentar para duas questões. Em primeiro lugar, é necessário reconhecer que a linguagem coloquial está longe de nos servir, visto que não carrega a precisão usualmente esperada no universo da ciência. Assim, com os conceitos psicológicos que vêm da linguagem coloquial, frequentemente somos colocados diante de fenômenos muito diversos (e, aqui, não se trata da complexidade variável das relações envolvidas), por vezes em nada relacionados uns com os outros (a rigor, estamos diante de topografias verbais que sequer são o mesmo conceito - cf. Tourinho, 2007). Essa constatação implica que, ao tentar oferecer uma interpretação analítico-comportamental para um desses conceitos, será neces- sário deixar claro, desde o início, qual é exatamente o universo dos fenômenos que se pretende explicar. A segunda questão importante é que, uma vez definidos os fenômenos de interesse, será necessário ao analista do comportamento decompô-los em relações comportamentais nem sempre acessíveis com os instrumentos investigativos disponíveis. O exercício de refletir sobre como se definem depressão e ansiedade enquanto relações comportamentais, exemplificado acima, constitui apenas isso - um exercício de uma tarefa que, levada a cabo em situações concretas, requer muito mais do que a ideia de um continuum de complexidade definido por relações produzidas nos três níveis de variação e seleção. O desenvolvimento tecnológico na análise do comportamento, no que concerne aos problemas aqui examinados, constitui ainda um enorme desafio, cujo enfrentamento requer a associação de competências profissionais e acadêmicas.

\section{Referências}

Anderson, C. M., Hawkins, R. P., \& Scotti, J. R. (1997). Private events in behavior analysis: Conceptual basis and clinical relevance. Behavior Therapy, 28(1), 157-179.

Barbosa, C. (2004). Ansiedade: Possíveis intervenções na análise do comportamento. Em M. Z. S. Brandão, F. C. S Conte, F. C. Brandão, Y. K. Ingberman, V. M. Silva, \& S. O. Oliane (Orgs.), Sobre comportamento e cognição: Vol. 13. Contingências e metacontingências: Contextos sócioverbais e o comportamento do terapeuta (pp. 163-167). Santo André, SP: Esetec.

Bornstein, P. H. (1975). Innovation in technique: A group-based induced anxiety. Revista Mexicana de Analisis de la Conducta. 1(2), 299-301.

Bouton, M. E., Mineka, S., \& Barlow, D. H. (2001). A modern learning theory perspective on the etiology of panic disorder. Psychological Review, 108(1), 4-32.

Cavalcante, S. N. (1997). Notas sobre o fenômeno da depressão a partir de uma perspectiva analítico-comportamental. Psicologia Ciência e Profissão, 17(2), 2-12.

Coêlho, N. L. (2006). O conceito de ansiedade na análise do comportamento. (Dissertação de mestrado não publicada). Universidade Federal do Pará, Belém, PA. 
Cone, J. D. (1998). Hierarchical views of anxiety: What do they profit us? Behavior Therapy, 29(2), 325-332.

Donahoe, J. W., \& Palmer, D. C. (1994). Learning and complex behavior. Boston, MA: Allyn and Bacon.

Dougher, M. J., \& Hackbert, L. (1994). A behavioranalytic account of depression and a case report using acceptance-based procedures. The Behavior Analyst, 17(2), 321-334.

Eifert, G. H. (1984). The effects of language conditioning on various aspects of anxiety. Behavior Research and Therapy, 22(1), 13-21.

Eifert, G. H., \& Wilson, P. H. (1991). The triple response approach to assessment: A conceptual and methodological reappraisal. Behavior Research and Therapy, 29(3), 283-292.

Forsyth, J. P., Eifert, G. H., \& Thompson, R. N. (1996). Systematic alarms in fear conditioning II: An experimental methodology using $20 \%$ carbon dioxide inhalation as an unconditioning stimulus. Behavior Therapy, 27(3), 391-415.

Forsyth, J. P., \& Eifert, G. H. (1996a). Systematic alarms in fear conditioning I: A reappraisal of what is being conditioned. Behavior Therapy, 27(3), 441-462.

Forsyth, J. P., \& Eifert, G. H. (1996b). The language of feeling and the feeling of anxiety: Contributions of the behaviorisms toward understanding the function-altering effects of language. The Psychological Record, 46(4), 607-649.

Forsyth, J. P. (2000). A process-oriented behavioral approach to the etiology, maintenance, and treatment of anxiety - related disorders. Em M. J. Dougher (Ed), Clinical behavior analysis (pp. 153-180). Reno, NV: Context Press.

Friman, P. C., Wilson, K. G., \& Hayes, S. C. (1998). Behavior analysis of private events is possible, progressive, and nondualistic: A response to Lamal. Journal of Applied Behavior Analysis, 31(4), 707-708.

Hayes, L. J. (1994). Thinking. Em S. C. Hayes, L. J. Hayes, M. Sato, \& K. Ono (Eds.), Behavior analysis of language and cognition (pp. 149164). Reno, NV: Context Press.

Hopko, D. R., McNaiel, D. W., Zvolensky, M. J., \& Eifert, G. H. (2001). The relation between anxiety and skill in performance-based anxiety disorders: A behavioural formulation of social phobia. Behavior Therapy, 32(1), 185-207.

Hunziker, M. H. L. (2001). O desamparo aprendido e a análise funcional da depressão. Em D. R. Zamignani (Org.), Sobre comportamento e cognição: Vol. 3. A aplicação da análise do comportamento e da terapia cognitivo-comportamental no hospital geral e nos transtornos psiquiátricos (pp. 143-151) Santo André, SP: Esetec.

Hunziker, M. H. L. (2005). O desamparo aprendido revisitado: Estudos com animais. Psicologia: Teoria e Pesquisa, 21(2), 131-139.

Jones, K. M., \& Friman, P. C. (1999). A case study of behavioral assessment and treatment of insect phobia. Journal of Applied Behavior Analysis, 32(1), 95-98.

Kanter, J. W., Callaghan, G. M., Landes, S. J, Busch, A. M., \& Brown, K. R. (2004). Behavior analytic conceptualization and treatment of depression: Traditional models and recent advances. The Behavior Analyst Today, 5(3), 255-274.

Kantor, J. R., \& Smith, N. W. (1975). The reaction system. Em J. R. Kantor \& N. W. Smith (Eds.), The science of psychology: An interbehavioral survey (pp. 47-55). Chicago, IL: Principia Press.

Lejuez, C. W., Hopko, D. R., \& Hopko, S. D. (2001). A brief behavioral activation treatment for depression: Treatment manual. Behavior Modification, 25(2), 255-286.

Lejuez, C. W., O'Donnell, J., Wirth, O., Zvolensky, M. J., \& Eifert, G. H. (1998). Avoidance of $20 \%$ carbon dioxide-enriched air with humans. Journal of the Experimental Analysis of behavior, 70(1), 79-86.

Observer (1973). Comments and queries: Private data, raw feels, inner experience, and all that. The Psychological Record, 23, 83-85.

Observer (1981). Comments and queries: Concerning the principle of psychological privacy. The Psychological Record, 31, 227-232.

Oliveira, M. A., \& Duarte, A. M. M. (2004). Controle de respostas de ansiedade em universitários em situações de exposições orais. Revista Brasileira de Terapia Comportamental e Cognitiva, 6(2), 183-199.

Öst, L. G. (1987). Applied relaxation: Description of a coping technique and review of controlled 
studies. Behavior Research and Therapy, 25(5), 397-409.

Queiroz, P. P., \& Guilhardi, H. J. (2001). Identificação e análise de contingências geradoras de ansiedade: Caso clínico. Em H. J. Guilhardi, M. B. B. P. Madi, P. P., Queiroz, \& M. C. Scoz (Orgs.), Sobre comportamento e cognição: Vol. 7. Expondo a variabilidade (pp. 257-268). Santo André, SP: Esetec.

Reiss, S., Peterson, R. A., Gursky, D. M., \& McNally, R. J. (1986). Anxiety sensitivity, anxiety frequency and the prediction of fearfulness. Behavior Research and Therapy, 24(1), 1-8.

Sanger, D. J., \& Blackman, D. E. (1976). The effects of $\mathrm{d}$-amphetamine $\mathrm{o}$ the temporal control of operant responding in rats during a preshock stimulus. Journal of The Experimental Analysis of Behavior, 26(3), 369-378.

Santos, A. M. (2000). Modelo comportamental da ansiedade. Em R. R. Kerbauy (Org.), Sobre comportamento e cognição: Vol. 5. Conceitos, pesquisa e aplicação, a ênfase no ensinar, na emoção e no questionamento clínico (pp. 189-191). Santo André, SP: Esetec.

Seligman, M. E. P. (1992). On development, depression and death. New York, NY: Freeman. (Trabalho original publicado em 1975)

Silva, M. T. A. (1997). Modelos animais de ansiedade. Em D. R. Zamignani (Org). Sobre Comportamento e Cognição: Vol. 3. A aplicação da análise do comportamento e da terapia cognitivo-comportamental no hospital geral e nos transtornos psiquiátricos (pp. 91-96). Santo André, SP: Arbytes.

Skinner, B. F. (1945). The operational analysis of psychological terms. Psychological Review, 52, 270- 277/291-294.

Skinner, B. F. (1965). Science and human behavior. New York, NY: Free Press. (Trabalho original publicado em 1953)

Skinner, B. F. (1976). About behaviorism. New York, NY: Vintage Books. (Trabalho original publicado em 1974)

Skinner, B. F. (1992). Verbal behavior. Acton, MA: Copley. (Trabalho original publicado em 1957).
Torres, N. (2000). Ansiedade: O enfoque do behaviorismo radical respaldando procedimentos clínicos. Em R. C. Wielenska (Org). Sobre comportamento e cognição: Vol 6. Questionando e ampliando a teoria e as intervenções clínicas em outros contextos (pp. 228-238). Santo André, SP: Arbytes.

Tourinho, E. Z. (1997a). Eventos privados em uma ciência do comportamento. Em R. A. Banaco (Org.), Sobre comportamento e cognição: Vol. 1. Aspectos teóricos, metodológicos e de formação em análise do comportamento e terapia cognitivista (pp.174-187). Santo André, SP: Arbytes.

Tourinho, E. Z. (1997b). Evento privado: Função e limites do conceito. Psicologia: Teoria e Pesquisa. 13(2), 203-209.

Tourinho, E. Z. (1997c). O conceito de comportamento encoberto no behaviorismo radical de B. F. Skinner. Em R. A. Banaco (Org.), Sobre comportamento e cognição: Vol. 1. Aspectos teóricos, metodológicos e de formação em análise do comportamento e terapia cognitivista (pp. 217-229). Santo André, SP: Arbytes.

Tourinho, E. Z. (1997d). Privacidade, comportamento e o conceito de ambiente interno. Em Banaco, R. A. (Org.), Sobre Comportamento e Cognição: Vol. 1. Aspectos teóricos, metodológicos e de formação em análise do comportamento e terapia cognitivista ( pp. 217-229). Santo André, SP: Arbytes.

Tourinho, E. Z. (1999a). Eventos privados: O que, como e por que estudar. Em R. R. Kerbauy \& R. C. Wielenska (Orgs.). Sobre comportamento e cognição: Vol. 4. Psicologia comportamental e cognitiva: Da reflexão teórica à diversidade na aplicação (pp. 13-25). Santo André, SP: Esetec.

Tourinho, E. Z. (1999b). Privacidade, comportamento e o conceito de ambiente interno. Em R. A. Banaco (Org.), Sobre comportamento e cognição: Vol. 1. Aspectos teóricos, metodológicos $e$ de formação em análise do comportamento $e$ terapia cognitivista (pp. 217-229). Santo André, SP: Arbytes.

Tourinho, E. Z. (2006a). Subjetividade e relações comportamentais. (Tese de professor titular não publicada). Universidade Federal do Pará, Belém, PA. 
Tourinho, E. Z. (2006b). Private stimuli, covert responses and private events: Conceptual remarks. The Behavior Analyst, 29(1), 13-31.

Tourinho, E. Z. (2006c). Relações comportamentais como objeto da Psicologia: Algumas implicações. Interação em Psicologia, 10(1), 1-8.

Tourinho, E. Z. (2007). Conceitos científicos e eventos privados como resposta verbal. Interação em Psicologia, 11(1), 1-9.

Tyndall, I. T., Roche, B., \& James, J. E. (2004). The relation between stimulus function and equivalence class formation. Journal of the Experimental Analysis of Behavior, 81(3), 257266.

Viliers, P. A., \& Millenson, J. R. (1972). Concurrent performances: A baseline for the study of conditioned anxiety. Journal of the Experimental Analysis of Behavior, 18(2), 287-294.

Weiss, A. R., \& Evans, I. M. (1978). Process studies in language conditioning. I: Counterconditioning of anxiety by "calm" words. Journal of Behavior Therapy and Experimental Psychiatry, 9(2), 115119.

Wlazlo, Z., Hartwing, K. S., Hand, I., Kaiser, G., \& Münchau, N. (1990). Exposure in vivo vs social skills training for social phobia: Long-term outcome and differential effects. Behavior Research and Therapy, 28(3), 181-193.

Wolpe, J. (1981). The dichotomy between classical conditioned and cognitively learned anxiety. Journal of Behavior Therapy and Experimental Psychiatry, 12(1), 35-42.

Wolpe, J., \& Rowan, V. C. (1988). Panic disorder: A product of classical conditioning. Behavior Research and Therapy, 26(6), 441-450.

Woods, D. W., \& Miltenberger, R. G. (1996). Are persons with nervous habits nervous? A preliminary examination of habits function in a nonreferred population. Journal of Applied Behavior Analysis, 29(2), 259-261.

Zamignani, D. R., \& Banaco, R. A. (2005). Um panorama analítico-comportamental sobre os transtornos de ansiedade. Revista Brasileira de Terapia Comportamental e Cognitiva, 7(1), 77-92.

Zamignani, D. R., \& Vermes, J. S. (2003). Propostas analítico-comportamentais para o manejo de transtornos de ansiedade: Análise de casos clínicos. Em H. M. Sadi, \& N. M. S. Castro (Orgs).
Ciência do comportamento: Conhecer e avançar: Vol. 3 (pp. 117-135). Santo André, SP: Esetec. Zvolensky, M. J. Lejuez, C. W., \& Eifert, G. H. (1998). The role of offset control in anxious responding: An experimental test using repeated administrations of $20 \%$ carbon dioxide-enriched air. Behavior Therapy, 29(2), 193-209.

\section{Informações do artigo}

\section{História do artigo}

data de submissão em 28/03/2010 primeira decisão editorial em 25/05/2010 aceito para publicação em 24/07/2010 